\title{
What do dg-categories form?
}

\author{
Dmitry Tamarkin
}

\begin{abstract}
We introduce a homotopy 2-category structure on the collection of dg-categories, dgfunctors, and their derived transformations. This construction provides for a conceptual proof of Deligne's conjecture on Hochschild cochains.
\end{abstract}

\section{Introduction}

It is well known that categories form a 2-category: 1-arrows are functors and 2-arrows are their natural transformations.

In a similar way, dg-categories also form a 2-category: 1-arrows $A \rightarrow B$ are dg-functors; given a pair of dg-functors $F, G: A \rightarrow B$ one can define a complex of their natural transformations $\operatorname{hom}(F, G)$, which naturally generalizes the notion of a natural transformation in the usual setting. Thus, we can use $\operatorname{hom}(F, G)$ as the space of 2-arrows. (As usual, a dg-category is by definition a category enriched over the symmetric monoidal category of complexes over a fixed ground field $k$.)

However, this construction has a serious drawback: the spaces $\operatorname{hom}(F, G)$ are not homotopically invariant in any way. For example, let $W: B \rightarrow C$ be a weak equivalence of dg-categories, then we have a natural map

$$
\operatorname{hom}(F, G) \rightarrow \operatorname{hom}(W F, W G),
$$

which, in general, is not a quasi-isomorphism of complexes.

Drinfeld [Dri04] proposes another construction, in which the role of dg-functors $A \rightarrow B$ is played by $A^{\mathrm{op}} \times B$ bi-modules. By choosing an appropriate class of such bi-modules, one can achieve a good homotopy behavior. Unfortunately, this class does not contain identity functors $A \rightarrow A$, but only their resolutions which satisfy the properties of identity up to homotopies.

The goal of this paper is to provide for a homotopy invariant structure on the category of a dg-category which, on the one hand, would be as close to the 2-category structure as possible; on the other hand, this structure should be free of the above-mentioned drawbacks.

In order to achieve a homotopy invariant behavior, one has to pass to a derived version of the notion of a natural transformation between two functors. This can be done in a very well-known way (see $\S \S 3.0 .2$ and 3.1 ).

As is common in such situations, these derived transformations of functors cannot be composed as nicely as the usual transformations of functors, so they do not form a 2-category.

Our result is that, informally speaking, derived natural transformations form a certain homotopy version of a 2-category. Let us now sketch the idea of the notion of a homotopy 2-category, precise definitions are given in the main body of the paper.

A good starting point is to reformulate the definition of a dg-2-category as follows.

Received 8 February 2006, accepted in final form 24 November 2006. 2000 Mathematics Subject Classification 18D05, 18D50, 55U10, 55U40.

Keywords: differential graded category, 2-operad, multisimplicial set.

This journal is (C) Foundation Compositio Mathematica 2007. 


\section{TAMARKIN}

A small 2-category is:

(i) a set of objects $C$, a set of 1 -arrows $\operatorname{hom}(X, Y)$ for every pair $X, Y \in \mathcal{C}$; these data should form a usual category;

(ii) a complex of 2-arrows $\operatorname{hom}(F, G)$ for all 1-arrows $F, G \in \operatorname{hom}(A, B)$.

These data should have the following structure:

(iii) given objects $A_{0}, A_{1}, \ldots, A_{n}$ and 1 -arrows $F_{i j}: A_{i} \rightarrow A_{i+1}, i=0,1, \ldots, n-1 ; j=0,1, \ldots, m_{i}$, one should have a composition map

$$
c: \bigotimes_{i j} \operatorname{hom}\left(F_{i j} ; F_{i, j+1}\right) \rightarrow \operatorname{hom}(F, G)
$$

where $F, G: A_{0} \rightarrow A_{n}$,

$$
F=F_{n-1,0} F_{n-2,0} \cdots F_{10} F_{00}, \quad \text { and } \quad G=G_{n-1, m_{n-1}} G_{n-1, m_{n-2}} \cdots G_{1 m_{1}} G_{0 m_{0}} .
$$

There should be a certain coherence axiom stating that these compositions are closed under iterations. Instead of giving a precise formulation, let us consider an example as follows.

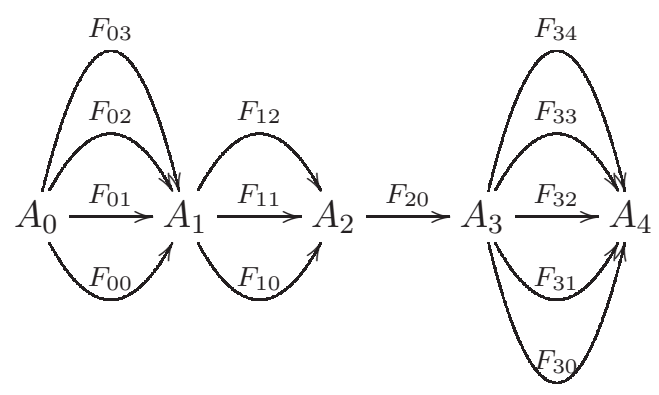

Let us split this picture into four sub-pictures as follows.

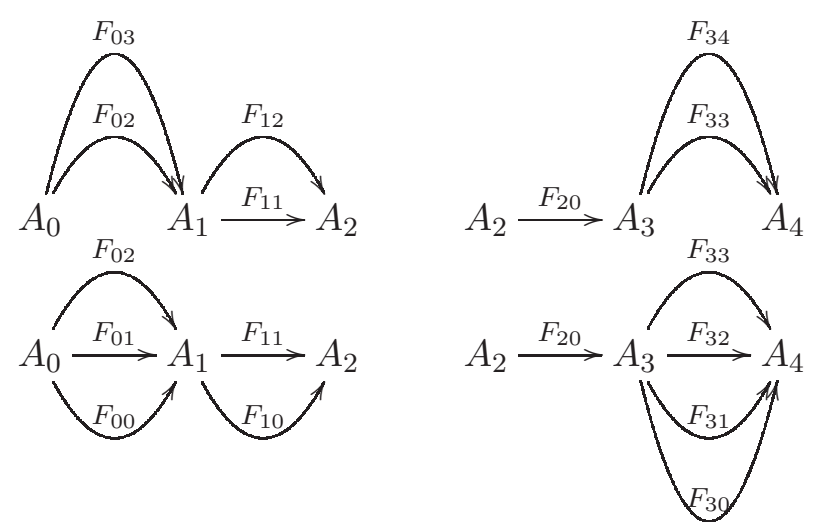

These sub-pictures yield the following composition maps:

$$
\begin{gathered}
\operatorname{hom}\left(F_{02}, F_{03}\right) \otimes \operatorname{hom}\left(F_{11}, F_{12}\right) \rightarrow \operatorname{hom}\left(F_{11} F_{02} ; F_{12} F_{03}\right) ; \\
\operatorname{hom}\left(F_{33}, F_{34}\right) \rightarrow \operatorname{hom}\left(F_{33} F_{20} ; F_{34} F_{20}\right) ; \\
\operatorname{hom}\left(F_{00}, F_{01}\right) \otimes \operatorname{hom}\left(F_{01}, F_{02}\right) \otimes \operatorname{hom}\left(F_{10}, F_{11}\right) \rightarrow \operatorname{hom}\left(F_{10} F_{00} ; F_{11} F_{02}\right) ; \\
\operatorname{hom}\left(F_{30}, F_{31}\right) \otimes \operatorname{hom}\left(F_{31}, F_{32}\right) \otimes \operatorname{hom}\left(F_{32}, F_{33}\right) \rightarrow \operatorname{hom}\left(F_{30} F_{20} ; F_{33} F_{20}\right) .
\end{gathered}
$$




\section{WHAT DO DG-CATEGORIES FORM?}

These maps can be composed with the composition map determined by the following 'quotientpicture':

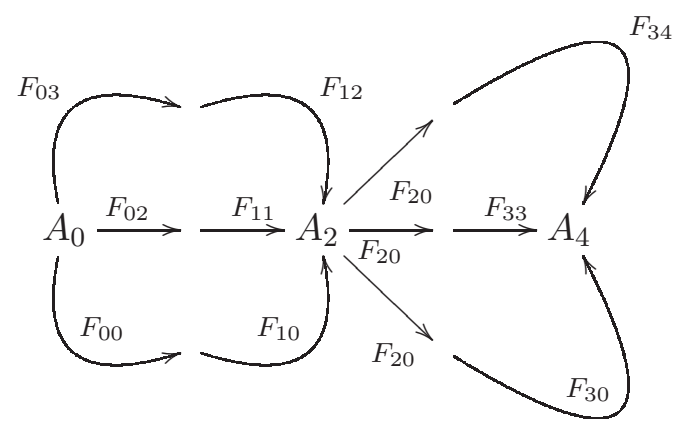

$$
\begin{aligned}
\operatorname{hom}\left(F_{11} F_{02} ; F_{12} F_{03}\right) \otimes \operatorname{hom}\left(F_{10} F_{00} ; F_{11} F_{02}\right) \otimes \operatorname{hom}\left(F_{30} F_{20} ;\right. & \left.F_{33} F_{20}\right) \operatorname{hom}\left(F_{33} F_{20} ; F_{34} F_{20}\right) \\
& \rightarrow \operatorname{hom}\left(F_{30} F_{20} F_{10} F_{00} ; F_{34} F_{20} F_{12} F_{03}\right)
\end{aligned}
$$

so as to get a map

$$
\begin{aligned}
& \operatorname{hom}\left(F_{00}, F_{01}\right) \otimes \operatorname{hom}\left(F_{00}, F_{01}\right) \otimes \operatorname{hom}\left(F_{01}, F_{02}\right) \otimes \operatorname{hom}\left(F_{02}, F_{03}\right) \otimes \operatorname{hom}\left(F_{10}, F_{11}\right) \\
& \otimes \operatorname{hom}\left(F_{11}, F_{12}\right) \otimes \operatorname{hom}\left(F_{30}, F_{31}\right) \otimes \operatorname{hom}\left(F_{31}, F_{32}\right) \otimes \operatorname{hom}\left(F_{32}, F_{33}\right) \otimes \operatorname{hom}\left(F_{33}, F_{34}\right) \\
& \rightarrow \operatorname{hom}\left(F_{30} F_{20} F_{10} F_{00} ; F_{34} F_{20} F_{12} F_{03}\right) .
\end{aligned}
$$

The coherence axiom then requires that the map that we have just constructed should coincide with the map (1) determined by the picture (2).

This definition can be homotopized in the following way (this is just a particular case of the notion of an algebra over a 2-operad from [Bat07]).

A homotopy 2-category is a collection of data (i), (ii) (as in the above definition) with (iii) modified as follows:

$\left(\right.$ iii $\left.^{\mathrm{h}}\right)$ for each collection of non-negative integers $m_{0}, m_{1}, m_{2}, \ldots, m_{n}$ there should be given a contractible complex $\mathcal{O}\left(m_{0}, m_{1}, \ldots, m_{n}\right)$ and a map

$$
c: \mathcal{O}\left(m_{0}, m_{1}, \ldots, m_{n}\right) \otimes \bigotimes_{i j} \operatorname{hom}\left(F_{i j} ; F_{i, j+1}\right) \rightarrow \operatorname{hom}(F, G),
$$

where the notation is the same as in (iii).

In order to formulate the coherence axiom we need some operad-like structure on the collection of complexes $\mathcal{O}\left(m_{0}, m_{1}, \ldots, m_{n}\right)$. Let us briefly discuss this structure.

First, with every picture $P$ as in (2), one naturally associates a complex $\mathcal{O}(P)$. (Example: for the picture from $(2), \mathcal{O}(P):=\mathcal{O}(3,2,0,4)$, where the numbers represent the numbers of arrows in each column of the picture); next, suppose that we have a subdivision of a picture $P$ into a number of sub-pictures $P_{1}, P_{2}, \ldots, P_{k}$ with the corresponding quotient-picture $Q$. (We do not define the precise meaning of these words hoping that the spirit can be garnered from the above example of a subdivision of the picture (2) into four sub-pictures (3) with the corresponding quotientpicture (4).)

We should then have a composition map

$$
\mathcal{O}(P) \otimes \mathcal{O}\left(P_{1}\right) \otimes \mathcal{O}\left(P_{2}\right) \otimes \cdots \otimes \mathcal{O}\left(P_{k}\right) \rightarrow \mathcal{O}(Q) .
$$

Having these maps, one can formulate the coherence axiom in this new setting in a natural way.

The maps (5) along with certain natural associativity properties constitute a so-called structure of a 2-operad [Bat07]; we will reproduce a precise definition. 


\section{TAMARKIN}

Our main result is that dg-categories form a homotopy 2-category in which objects are dgcategories, 1-arrows are functors, and the complexes of 2-arrows are defined using the derived version of the complex of natural transformations.

We conclude the paper with an observation that this result immediately implies that for every category $\mathcal{C}$, the complex $\operatorname{Rhom}\left(\operatorname{Id}_{C}, \operatorname{Id}_{C}\right)$ (the homotopy center of $\mathcal{C}$ ) is an algebra over the abovementioned 2-operad $\mathcal{O}$. A result from [Bat07] implies that an algebra structure over any contractible 2-operad (such as $\mathcal{O}$ ) implies a structure of an algebra over some resolution of the chain operad of little disks, thus yielding another proof of Deligne's conjecture on Hochschild cochains [KS00, Vor00, MS02, BF04].

The plan of the paper is as follows. We begin by defining a cosimplicial complex of natural transformations $\operatorname{hom}^{\bullet}(F, G)$ for every pair of dg-functors $F, G: A \rightarrow B$. By taking the realization, one obtains a complex $\operatorname{Rhom}(F, G):=\left|\operatorname{hom}^{\bullet}(F, G)\right|$ which we use as a replacement for the naive complex $\operatorname{hom}(F, G)$. Then, we introduce some combinatorics in order to describe the pictures such as (2). Next, we provide definitions of a 2-operad (which is equivalent to that in [Bat07]) and a homotopy 2-category. After that, we proceed to construct a homotopy 2-category of dgcategories. It turns out to be more convenient to start by constructing a certain structure on the cosimplicial complexes $\operatorname{hom}^{\bullet}(F, G)$, without passing to the realization. This structure will be given in terms of a collection of certain multisimplicial sets so that one can study them using some topology. Finally, using the realization functors, we convert this structure into a homotopy 2 -category structure in which the complexes of 2-arrows are $\operatorname{Rhom}(F, G)$. We conclude by showing that this result coupled with Batanin's theorem on contractible 2-operads readily implies Deligne's conjecture.

\section{Conventions and notation}

Any finite non-empty totally ordered set will be called an ordinal.

Given a non-negative integer $n$, we denote by $[n]$ the ordinal $\{0<1<\cdots<n\}$.

Given an ordinal $I$ we denote its minimum by $m_{I}$ and its maximum by $M_{I}$.

Denote by $\vec{I}$ the set of all pairs $\overrightarrow{\imath \jmath}$, where $i, j \in I$ and $j$ is the immediate successor of $i$. We have an induced total order on $\vec{I}$, but $\vec{I}$ may be empty. We have natural projections $s, t: \vec{I} \rightarrow I$, $s(\overrightarrow{\imath \jmath})=i$, and $t(\overrightarrow{\imath \jmath})=j$.

Given $a, b \in I, a \leqslant b$, we define the interval $[a, b] \subset I$ in the usual way.

A monotone (that is, non-decreasing) map of ordinals $f: I \rightarrow J$ will be called dominant if $f\left(m_{I}\right)=m_{J}$ and $f\left(M_{I}\right)=M_{J}$.

Ordinals and their monotone dominant maps form a category. In [Joy97], this category is called the category of 1-disks and its opposite is identified with the category $\Delta$ of ordinals and their monotone maps.

\section{Functors between dg-categories and their natural transformations}

3.0.1 Let $A$ be a dg-category. Let $X: I \rightarrow A$ be a family of objects in $A$ indexed by an ordinal $I$. Set

$$
A(X):=\bigotimes_{\vec{\imath} \in \vec{I}} A(s(\vec{\imath}) ; t(\vec{\imath})) .
$$

If $I$ is a one-element ordinal, we set $A(X)=k$.

Given a dominant monotone map $k: J \rightarrow I$, we have a natural map

$$
k^{*}: A(X) \rightarrow A(X \circ k) .
$$




\section{WHAT DO DG-CATEGORIES FORM?}

3.0.2 Definition of the cosimplicial complex of natural transformations. Let $A, B$ be small dg-categories and $F, G: A \rightarrow B$ functors.

Let $I$ be a finite non-empty totally ordered set. Set

$$
\operatorname{hom}^{I}(F ; G):=\prod_{X: I \rightarrow A} \operatorname{hom}_{k}\left(A(X) ; \operatorname{hom}_{B}\left(F\left(X\left(m_{I}\right)\right) ; G\left(X\left(M_{I}\right)\right)\right)\right) \text {. }
$$

Let $I^{\prime}$ be obtained from $I$ by adding two elements $m^{\prime}, M^{\prime}$ such that $m^{\prime}<I<M^{\prime}$. Let $X^{\prime}: I^{\prime} \rightarrow A$ and let $X$ be the restriction of $X^{\prime}$ onto $I$. Then we have

$$
A\left(X^{\prime}\right) \cong A\left(X\left(M_{I}\right) ; X^{\prime}\left(M^{\prime}\right)\right) \otimes A(X) \otimes A\left(X^{\prime}\left(m^{\prime}\right) ; X\left(m_{I}\right)\right) .
$$

We have a natural map

$$
\operatorname{hom}^{I}(F, G) \rightarrow \operatorname{hom}^{I^{\prime}}(F, G)
$$

such that the chain $\Phi \in \operatorname{hom}^{I}(F, G)$ is mapped into a chain $\Phi^{\prime}$ according to the rule

$$
\Phi^{\prime}(\omega \otimes U \otimes \alpha)=G(\omega) \circ \Phi(U) \circ F(\alpha),
$$

where $\omega \in A\left(X(M), X^{\prime}\left(M^{\prime}\right)\right), U \in A(X), \alpha \in A\left(X^{\prime}\left(m^{\prime}\right), X(m)\right)$, and we use the identification (6).

3.0.3 Cosimplicial structure. Let $\Delta$ be the category of ordinals and their monotone (that is, non-decreasing) maps. We are going to endow the collection of spaces $\mathbf{h o m}^{I}(F, G)$ with a structure of the functor

$$
\Delta \rightarrow \text { complexes. }
$$

Let $\sigma: I \rightarrow J$ be a monotone map. Define a map

$$
\sigma_{*}: \operatorname{hom}^{I}(F, G) \rightarrow \operatorname{hom}^{J}(F, G)
$$

as follows.

Let $\sigma^{\prime}: I^{\prime} \rightarrow J$ be the extension of $\sigma$ which sends $m^{\prime}$ and $M^{\prime}$ to the minimum and the maximum of $J$, respectively.

Set $\sigma_{*} \Phi(U):=\Phi^{\prime}\left(\left(\sigma^{\prime}\right)^{*} U\right)$, where $U \in A(X)$ for some $X: J \rightarrow A$. In this way we get the desired cosimplicial structure.

\subsection{Total complex of a cosimplicial complex}

It is well known that given a cosimplicial complex, one can produce its total complex by applying alternated sums of co-face maps.

We use a slightly different definition of this total complex. Let $I$ be an ordinal and let $\Delta^{I}$ be the simplex whose vertices are labeled by $I$. Let $C_{*}\left(\Delta^{I}\right)$ be its reduced chain complex. Let $\mathcal{S}^{*}(I):=C_{-*}\left(\Delta^{I}\right)$. It is clear that $\mathcal{S}^{*}(\bullet)$ is a cosimplicial complex (here $\bullet$ stands for the 'cosimplicial' argument). We denote this cosimplicial complex simply by $\mathcal{S}$.

Given an arbitrary cosimplicial complex $K$ we can form a complex hom $\Delta(\mathcal{S}, K)$ which will also be denoted by $|K|$. In this formula, hom $\Delta$ means 'enriched hom-functor in the category of cosimplicial complexes'.

Thus, we can construct a complex $\left|\operatorname{hom}^{\bullet}(F, G)\right|$ which will be denoted by $\operatorname{Rhom}(F, G)$.

3.1.1 We also have a 'naive' notion of the complex of natural transformations of two functors. Indeed, given a pair of functors $F, G: A \rightarrow B$, define the complex

$$
\operatorname{hom}(F, G)
$$




\section{TAMARKIN}

as the equalizer of the diagram

$$
\operatorname{hom}(F, G) \longrightarrow \operatorname{hom}^{[0]}(F, G) \underset{d_{0}}{\stackrel{d_{1}}{\longrightarrow}} \operatorname{hom}^{[1]}(F, G)
$$

where $d_{0}, d_{1}$ are the co-face maps.

We can define a constant cosimplicial complex

$$
\operatorname{hom}^{\bullet}(F, G)
$$

where

$$
\operatorname{hom}^{I}(F, G):=\operatorname{hom}(F, G) .
$$

We then have a cosimplicial map

$$
\operatorname{hom}^{\bullet}(F, G) \rightarrow \operatorname{hom}^{\bullet}(F, G) .
$$

\section{Some combinatorics}

We want to find an algebraic structure naturally possessed by complexes $\operatorname{Rhom}(F, G)$. This structure will be given in terms of a family of multilinear maps between these complexes and some relations between them. In order to formulate this structure we need some combinatorics.

\subsection{Combinatorial data}

4.1.1 2-ordinals, 2-trees. By definition, a 2-ordinal $\mathcal{U}$ is a collection of the following data: ${ }^{1}$

(i) an ordinal $\mathcal{C}_{\mathcal{U}}$;

(ii) for each $\vec{c} \in \overrightarrow{\mathcal{C}}_{\mathcal{U}}$, an ordinal $\mathcal{F}_{\mathcal{U}, \vec{c}}$.

2-ordinals are meant to represent pictures of the following type.

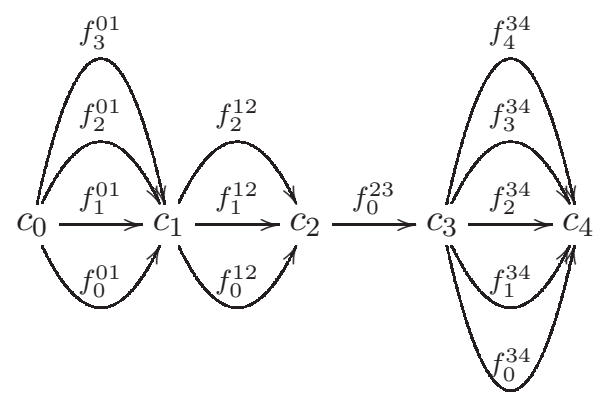

This picture corresponds to the following 2-ordinal:

(i) $\mathcal{C}=\left\{c_{0}<c_{1}<c_{2}<c_{3}<c_{4}\right\}$;

(ii) $\mathcal{F}_{\overrightarrow{c_{0} c 1}}=\left\{f_{0}^{01}<f_{1}^{01}<f_{2}^{01}<f_{3}^{01}\right\}$;

(iii) $\mathcal{F}_{\overrightarrow{c_{1} c_{2}}}=\left\{f_{0}^{12}<f_{1}^{12}<f_{2}^{12}\right\}$;

(iv) $\mathcal{F}_{\overrightarrow{c_{2} c_{3}}}=\left\{f_{0}^{23}\right\}$;

(v) $\mathcal{F}_{\overrightarrow{c_{3} c_{4}}}=\left\{f_{0}^{34}<f_{1}^{34}<\cdots<f_{4}^{34}\right\}$

A picture of this type can be drawn for any 2-ordinal in the obvious way.

We denote

$$
\overrightarrow{\mathcal{F}}_{\mathcal{U}}:=\bigsqcup_{\vec{c} \in \overrightarrow{\mathcal{C}}} \overrightarrow{\mathcal{F}}_{\mathcal{U}, \vec{c}}
$$

\footnotetext{
${ }^{1}$ An equivalent notion is introduced in [Str00], where it is called 'a globular 2-cardinal'.
} 


\section{WHAT DO DG-CATEGORIES FORM?}

Elements of this set can be visualized as 2-cells in the picture corresponding to $\mathcal{U}$. We then have an obvious monotone map of finite totally ordered sets:

$$
\pi: \overrightarrow{\mathcal{F}}_{\mathcal{U}} \rightarrow \overrightarrow{\mathcal{C}}_{\mathcal{U}}
$$

According to [Bat07], let us call any map of finite totally ordered sets a two-stage tree or, in abbreviated form, a 2-tree.

We denote 2-trees by one letter, say $t$. We refer to the elements of $t$ as

$$
\pi_{t}: \overrightarrow{\mathcal{F}}_{t} \rightarrow \overrightarrow{\mathcal{C}}_{t}
$$

We have shown how, given a 2-ordinal $\mathcal{U}$, one constructs a 2 -tree $\pi_{U}: \overrightarrow{\mathcal{F}}_{\mathcal{U}} \rightarrow \overrightarrow{\mathcal{C}}_{\mathcal{U}}$. Denote this 2-tree by $t_{\mathcal{U}}$, so that

$$
\begin{gathered}
\pi_{t_{\mathcal{U}}}:=\pi_{\mathcal{U}}, \quad \overrightarrow{\mathcal{F}}_{t_{\mathcal{U}}}:=\overrightarrow{\mathcal{F}}_{\mathcal{U}}, \\
\overrightarrow{\mathcal{C}}_{t_{\mathcal{U}}}:=\overrightarrow{\mathcal{C}}_{\mathcal{U}} .
\end{gathered}
$$

It is clear that a 2-ordinal is defined up to a canonical isomorphism by its 2 -tree. ${ }^{2}$

4.1.2 Given a 2-ordinal $\mathcal{U}$ we can construct a strict 2-category [ $\mathcal{U}]$, the universal category among the 2-categories $V$ possessing the following properties:

(i) $\mathrm{Ob} V=\mathcal{C}$;

(ii) there are fixed maps $\overrightarrow{\mathcal{F}_{\overrightarrow{c_{1} c_{2}}}} \rightarrow \mathbf{O b}_{\operatorname{Ohom}_{V}}\left(c_{1}, c_{2}\right)$ for all $\overrightarrow{c_{1} c_{2}} \in \overrightarrow{\mathcal{C}}$; for an $f \in \overrightarrow{\mathcal{F}_{\vec{c} c_{2}}}$ we denote by the same symbol the corresponding object in $\operatorname{hom}_{V}\left(c_{1}, c_{2}\right)$;

(iii) for each $\overrightarrow{f_{1} f_{2}} \in \overrightarrow{\mathcal{F}} \underset{c_{1} c_{2}}{\longrightarrow}$ we have a fixed element in $\operatorname{hom}_{\operatorname{hom}_{V}\left(c_{1}, c_{2}\right)}\left(f_{1}, f_{2}\right)$.

This 2-category has a clear meaning in terms of the picture (7).

Objects are $c_{0}, c_{1}, \ldots$; the space of maps $c_{i} \rightarrow c_{j}$ is non-empty if and only if $c_{i} \leqslant c_{j}$, in which case an arrow $c_{i} \rightarrow c_{j}$ is just a directed path from $c_{i}$ to $c_{j}$; let us define a partial order on the space of such paths by declaring that one path is less than or equal to another if and only if the former lies below the latter. We then have a category structure on hom $\left(c_{i}, c_{j}\right)$ produced by the just-defined poset of paths (each arrow goes from a smaller object to a greater one).

Here is a more formal description. Given $c, c^{\prime} \in \mathcal{C}$, we have:

(i) $\operatorname{hom}\left(c, c^{\prime}\right)=\emptyset$ if $c>c^{\prime}$;

(ii) $\operatorname{hom}(c, c)=\left\{\operatorname{Id}_{c}\right\}$;

(iii) if $c<c^{\prime}$, then

$$
\operatorname{Obhom}\left(c, c^{\prime}\right):=\prod_{\vec{c} \in \overrightarrow{\left[c, c^{\prime}\right]}} \mathcal{F}_{\vec{c}} .
$$

Given $f^{1}, f^{2} \in \operatorname{hom}\left(c, c^{\prime}\right)$,

$$
f^{k}=\left\{f_{\vec{c}}^{k}\right\}_{\vec{c} \in\left[\overrightarrow{\left.c_{1} c_{2}\right]}\right.}, \quad k=1,2,
$$

we have a unique arrow $f_{1} \rightarrow f_{2}$ if and only if

$$
f_{\vec{c}}^{1} \leqslant f_{\vec{c}}^{2}
$$

for all $\vec{c} \in \overrightarrow{\left[c_{1} c_{2}\right]}$. Thus, the set $\operatorname{hom}\left(c_{1}, c_{2}\right)$ is partially ordered and has the least and the greatest elements.

\footnotetext{
${ }^{2}$ This identification of 2-ordinals and 2-trees is introduced in [Bat98], where it is called the $(-) *$-construction.
} 


\section{TAMARKIN}

4.1.3 We will often need a special 2-ordinal called a globe and denoted by globe. We define globe by

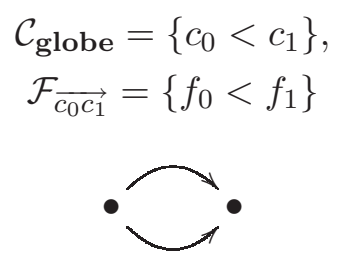

Any 2-ordinal isomorphic to globe will also be called a globe.

4.1.4 Balls in a 2-ordinal. Given a 2-ordinal $\mathcal{U}$ define a ball in $\mathcal{U}$ as any 2-ordinal $\mathcal{U}^{\prime}$ of the form:

(i) $\mathcal{C}_{\mathcal{U}^{\prime}}$ is an interval in $\mathcal{C}_{\mathcal{U}}$;

(ii) for each $\vec{c} \in \overrightarrow{\mathcal{C}}_{\mathcal{U}^{\prime}}, \mathcal{F}_{\mathcal{U}^{\prime}, \vec{c}}$ is an interval in $\mathcal{F}_{\mathcal{U}, \vec{c}}$.

We then see that

$$
\left[\mathcal{U}^{\prime}\right] \subset[\mathcal{U}]
$$

is a full subcategory.

The set of all balls in $\mathcal{U}$ is partially ordered; each minimal ball is a globe; the set of all of these minimal balls is naturally identified with $\overrightarrow{\mathcal{F}}_{\mathcal{U}}$.

4.1.5 We write $[\mathcal{U}]_{1}$ for the underlying usual category of $\mathcal{U}$.

4.1.6 Maps of 2-ordinals. Let $\mathcal{U}, \mathcal{V}$ be 2-ordinals. $A \operatorname{map}^{3} P: \mathcal{U} \rightarrow \mathcal{V}$ is a 2-functor $[P]:[\mathcal{V}] \rightarrow$ $[\mathcal{U}]$ satisfying:

(i) the induced map $[P]: \mathcal{C}_{\mathcal{V}} \rightarrow \mathcal{C}_{\mathcal{U}}$ is dominant (that is, monotone and preserves the minima and the maxima);

(ii) for all $c_{1}<c_{2}, c_{1}, c_{2} \in \mathcal{C}_{V}$ the induced map

$$
\operatorname{hom}_{[\mathcal{V}]}\left(c_{1}, c_{2}\right) \rightarrow \operatorname{hom}_{[\mathcal{U}]}\left(P\left(c_{1}\right), P\left(c_{2}\right)\right)
$$

preserves the least and the greatest elements.

With this definition of a map, 2-ordinals form a category. Any globe is a terminal object in this category.

4.1.7 Inverse images of balls. Let $P: \mathcal{U} \rightarrow \mathcal{V}$ be a map of 2-ordinals and $\mathcal{V}^{\prime} \subset \mathcal{V}$ a ball in $\mathcal{V}$. Define $P^{-1} \mathcal{V}^{\prime}=: \mathcal{U}^{\prime}$ as a unique ball in $\mathcal{U}$ satisfying. There exists a map of 2-ordinals $P^{\prime}: \mathcal{U}^{\prime} \rightarrow \mathcal{V}^{\prime}$ fitting into a commutative diagram:

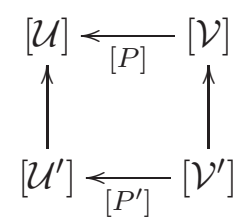

with the vertical arrows being the natural inclusions.

\footnotetext{
${ }^{3}$ See [Ber02] for the definition of an equivalent concept of a covering map of $n$-trees.
} 


\section{WHAT DO DG-CATEGORIES FORM?}

Consider the following pictorial example. Let $\mathcal{U}$ be the same ordinal as in (7):

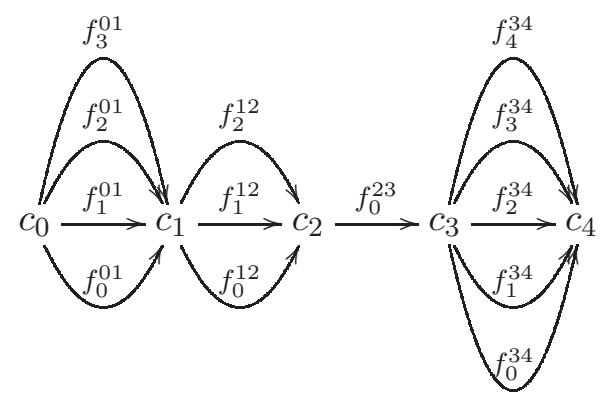

and let $\mathcal{V}$ be defined by the following picture.

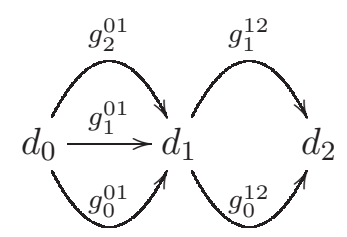

Consider a map $P: \mathcal{U} \rightarrow \mathcal{V}$ such that the corresponding map $[P]:[\mathcal{V}] \rightarrow[\mathcal{U}]$ is as follows:

$$
\begin{gathered}
{[P] d_{0}=c_{0}, \quad[P] d_{1}=c_{2}, \quad[P] d_{2}=c_{4},} \\
{[P] g_{0}^{01}=f_{0}^{12} f_{0}^{01}, \quad[P] g_{1}^{01}=f_{1}^{12} f_{0}^{01}, \quad[P] g_{2}^{01}=f_{2}^{12} f_{3}^{01},} \\
{[P] g_{0}^{12}=f_{0}^{34} f_{0}^{23},} \\
{[P] g_{1}^{12}=f_{4}^{34} f_{0}^{23} .}
\end{gathered}
$$

Let us label globes in $\mathcal{V}$ by $I, I I, I I I$ as shown in the following picture.

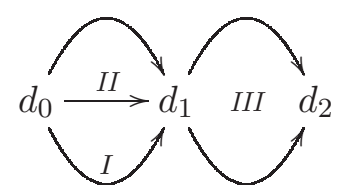

The preimages of these globes are then as follows.
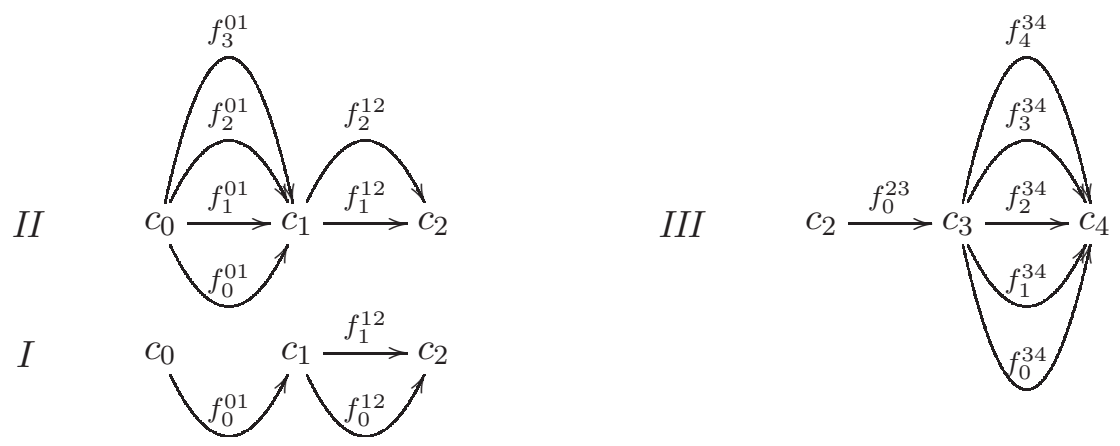


\section{TAMARKIN}

4.1.8 Maps of the corresponding 2-trees. According to Batanin, we define a map of 2-trees $P: t_{1} \rightarrow t_{2}$ as a commutative diagram

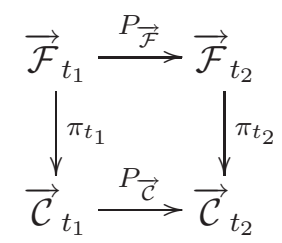

with the arrow $P_{\overrightarrow{\mathcal{C}}}$ being monotone and the arrow $P_{\overrightarrow{\mathcal{F}}}$ being monotone on each fiber of $\pi_{t_{1}}$ (i.e. on each subset $\pi_{t_{1}}(c) \subset \overrightarrow{\mathcal{F}}_{t_{1}}$ for each $\left.c \in \overrightarrow{\mathcal{C}}_{t_{1}}\right)$.

4.1.9 A map of ordinals $\mathcal{U} \rightarrow \mathcal{V}$ naturally induces a map of the sets of two-cells:

$$
\overrightarrow{\mathcal{F}}_{\mathcal{U}} \rightarrow \overrightarrow{\mathcal{F}}_{V}
$$

it is not hard to see that this map lifts to a map of the corresponding 2-trees. We now give a formal definition of this map.

Given a map of 2-ordinals $P: \mathcal{U} \rightarrow \mathcal{V}$, we define an induced map of the associated 2-trees

$$
P^{t}: t_{\mathcal{U}} \rightarrow t_{V}
$$

as follows.

(i) Define the map

$$
P_{\overrightarrow{\mathcal{C}}}^{t}: \overrightarrow{\mathcal{C}}_{\mathcal{U}} \rightarrow \overrightarrow{\mathcal{C}}_{V}
$$

For $\overrightarrow{c_{1} c_{2}} \in \overrightarrow{\mathcal{C}}_{U}$ we set $P_{\overrightarrow{\mathcal{C}}}\left(\overrightarrow{c_{1} c_{2}}\right)=\overrightarrow{d_{1} d_{2}}$ if and only if

$$
\left[c_{1} c_{2}\right] \subset\left[[P]\left(d_{1}\right),[P]\left(d_{2}\right)\right] .
$$

(ii) Given a globe $\vec{f} \in \overrightarrow{\mathcal{F}}_{\mathcal{U}}$, define its image $P_{\overrightarrow{\mathcal{F}}}^{t}(\vec{f})=: \vec{g}$ in $\overrightarrow{\mathcal{F}}_{\mathcal{V}}$ as a unique globe such that the ball $P^{-1} \vec{g}$ contains $\vec{f}$.

4.1.10 We see that in this way we have constructed a category of 2-ordinals, a category of 2-trees, and a functor between them; as shown in [Ber02], this functor is an equivalence.

4.1.11 Diagrams. Given a 2-ordinal $\mathcal{U}$ and a category $\mathcal{C}$, a $\mathcal{U}$-diagram in $\mathcal{C}$ is a functor

$$
\mathcal{D}:[U]_{1} \rightarrow \mathcal{C} .
$$

Given a map of 2-ordinals $P: \mathcal{U} \rightarrow \mathcal{V}$ and a $\mathcal{U}$-diagram $\mathcal{D}$, it naturally restricts to produce $P^{-1} \vec{f}$ diagrams $\left.\mathcal{D}\right|_{P^{-1} \vec{f}}$, where $\vec{f} \in \overrightarrow{\mathcal{F}}_{\mathcal{V}}$, and a $\mathcal{V}$ diagram $P_{*} \mathcal{D}$. These induced diagrams come from the obvious functors

$$
\begin{aligned}
& {\left[P^{-1} \vec{f}\right] \hookrightarrow[\mathcal{U}],} \\
& {[P]:[\mathcal{V}] \rightarrow[U] .}
\end{aligned}
$$

\section{The 2-operads and their algebras}

We are going to adjust the notions of an operad and an algebra over an operad so that they work in our setting. In the usual setting, given an operad, we define its action on a complex; in our situation, instead of one complex, we have a family of complexes: a $\operatorname{complex} \operatorname{Rhom}(F, G)$ for each 
globe formed by the pair of dg-categories $A, B$ and a pair of dg-functors $F, G: A \rightarrow B$. We abstract this situation by introducing a notion of a $\mathcal{C}$-complex. Next, following [Bat07], we define the notion of a 2 -operad, and, lastly, the notion of a structure of an $\mathcal{O}$-algebra on a $\mathcal{C}$-complex, where $\mathcal{O}$ is a 2-operad.

Using these notions, we will be able to formulate the definition of a homotopy 2-category as an algebra structure over a contractible 2-operad.

\section{$5.1 \mathcal{C}$-complexes and their tensor products}

We fix a small category in sets $\mathcal{C}$. Let $\mathcal{C}_{0}$ be the set of objects in $\mathcal{C}$ and $\mathcal{C}_{1}$ be the set of its arrows. Let $s, t: \mathcal{C}_{1} \rightarrow \mathcal{C}_{0}$ be the source and target maps.

We define a globe in $\mathcal{C}$ as any globe-diagram in $\mathcal{C}$ (that is, a pair of objects in $\mathcal{C}$ and a pair of arrows between these objects).

Let $\mathcal{C}_{2}$ be the set of all globes in $\mathcal{C}$. We have obvious maps

$$
s^{\prime}, t^{\prime}: \mathcal{C}_{2} \rightarrow \mathcal{C}_{1}
$$

given a globe $g$

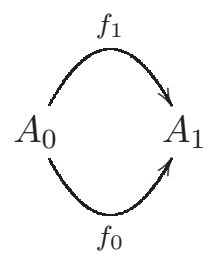

where $A_{0}, A_{1} \in \mathcal{C}, f_{0}, f_{1}: A_{0} \rightarrow A_{1}$, we set $s^{\prime}(g)=f_{0}, t^{\prime}(g)=f_{1}$. It is clear that

$$
s s^{\prime}=t s^{\prime}, \quad s t^{\prime}=t t^{\prime},
$$

and $\mathcal{C}_{2}$ is the terminal object in the category of all sets $Z$ endowed with maps $s^{\prime}, t^{\prime}: Z \rightarrow \mathcal{C}_{1}$ satisfying (8).

5.1.1 We define a $\mathcal{C}$-complex as a family of complexes parameterized by $\mathcal{C}_{2}$.

5.1.2 Tensor product of $\mathcal{C}$-complexes. Given a 2-ordinal $\mathcal{U}$ and a $\overrightarrow{\mathcal{F}}_{\mathcal{U}}$-family of $\mathcal{C}$-complexes $\mathcal{K}=\left\{K_{\vec{f}}\right\} \vec{f} \in \overrightarrow{\mathcal{F}}_{\mathcal{C}}$, we define a new $\mathcal{C}$-complex

$$
\bigotimes^{\mathcal{U}}:=\bigotimes_{\vec{f} \in \overrightarrow{\mathcal{F}}_{\mathcal{U}}}^{\mathcal{U}} K_{\vec{f}}
$$

as follows.

Pick a globe $g \in C_{2}$ and define

$$
\left(\bigotimes^{\mathcal{U}}\right)_{g}:=\bigoplus_{\mathcal{D} \mid p_{*} \mathcal{D}=g} \bigotimes_{\vec{f} \in \overrightarrow{\mathcal{F}}_{\mathcal{U}}} K_{(\mathcal{D} \mid \vec{f})},
$$

where $p: \mathcal{U} \rightarrow$ globe is the terminal map, $\mathcal{D}$ is any $\mathcal{U}$-diagram in $\mathcal{C}$ with $p_{*} \mathcal{D}=g$, and $\overrightarrow{\mathcal{F}}_{\mathcal{U}}$ is identified with the set of globes in $\mathcal{U}$ so that $\left.\mathcal{D}\right|_{f}$ is a globe in $\mathcal{C}$. 


\section{TAMARKIN}

5.1.3 Given a map of 2-ordinals $\mathcal{U} \rightarrow \mathcal{V}$, we have a canonical isomorphism

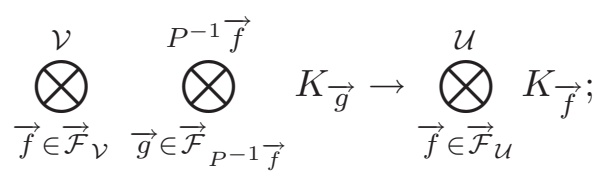

we call this isomorphism a constraint.

Given a chain of maps of 2-ordinals $\mathcal{U} \rightarrow \mathcal{V} \rightarrow \mathcal{W}$, we get the associativity property of this constraint.

This can be reformulated so that the category of $\mathcal{C}$-complexes becomes a category with two monoidal structures such that one of them distributes over the other but we will not need it in this paper.

5.1.4 Fix a set Chrom to be called the set of colors. Let us also fix family of $\mathcal{C}$-complexes $\mathcal{K}:=\left\{K_{\chi}\right\}_{\chi \in \text { Chrom }}$.

Let $\mathcal{U}$ be a 2-ordinal. Define $a$ Chrom-coloring $\chi$ of $\mathcal{U}$ as a prescription of a color $c_{\vec{f}}^{\chi} \in$ Chrom for each $\vec{f} \in \overrightarrow{\mathcal{F}}_{\mathcal{U}}$ and an additional color $c^{\chi} \in$ Chrom.

A 2-ordinal endowed with a coloring will be called a colored 2-ordinal. Given a colored 2-ordinal $\mathcal{U}^{\prime}:=(\mathcal{U}, \chi)$, we have a complex

$$
\operatorname{full}_{\mathcal{K}}\left(\mathcal{U}^{\prime}\right):=\operatorname{hom}\left(\left[\underset{\vec{f} \in \overrightarrow{\mathcal{F}}_{\mathcal{U}}}{\mathcal{U}} K_{c_{\vec{f}}^{\chi}}\right] ; K_{c \chi}\right) .
$$

These complexes form an algebraic structure called a colored 2-operad. We now define this notion.

\subsection{Colored 2-operads}

We need a notion of a map of colored 2-ordinals. Let $\mathcal{U}^{\prime}=\left(\mathcal{U}, \chi_{\mathcal{U}}\right) ; \mathcal{V}^{\prime}=(\mathcal{V}, \chi \mathcal{V})$ be colored ordinals. We say that we have a map $P^{\prime}: \mathcal{U}^{\prime} \rightarrow \mathcal{V}^{\prime}$ if:

(i) we are given a map $P: \mathcal{U} \rightarrow \mathcal{V}$ of 2-ordinals;

(ii) $c^{\chi \mathcal{U}}=c^{\chi \mathcal{V}}$.

Given a globe $\vec{f} \in \overrightarrow{\mathcal{F}} \mathcal{V}$, we then have a natural coloring $\chi^{\vec{f}}$ on $P^{-1} \vec{f}$ : we set $c^{\chi^{\vec{f}}}=c \underset{f}{\chi \mathcal{V}},\left(c^{\chi \vec{f}}\right)_{\vec{g}}:=$ $\left(c^{\chi \mathcal{U}}\right) \vec{g}$.

5.2.1 Definition of a colored 2-operad. A Chrom-colored operad $\mathcal{O}$ is a collection of the following data:

(i) a functor $\mathcal{O}$ from the isomorphism groupoid of the category of colored 2-ordinals to the category of complexes;

(ii) given a map $P: \mathcal{U}^{\prime} \rightarrow \mathcal{V}^{\prime}$ of colored ordinals, set

$$
\mathcal{O}(P):=\bigotimes_{\vec{f} \in \overrightarrow{\mathcal{F}}_{\mathcal{V}}} \mathcal{O}\left(P^{-1} \vec{f}\right)
$$

we then should have a map

$$
{ }^{\circ}: \mathcal{O}(P) \otimes \mathcal{O}\left(\mathcal{V}^{\prime}\right) \rightarrow \mathcal{O}\left(\mathcal{U}^{\prime}\right)
$$

Axioms. The first axiom asks for covariance of the map $\circ_{P}$ under isomorphisms of 2-ordinals. 


\section{WHAT DO DG-CATEGORIES FORM?}

In order to formulate the next axiom, note that given a chain of maps of colored 2-ordinals

$$
\mathcal{U} \stackrel{P}{\rightarrow} \mathcal{V} \stackrel{Q}{\rightarrow} \mathcal{W}
$$

we have a natural map

$$
\circ(Q, P): \mathcal{O}(P) \otimes \mathcal{O}(Q) \rightarrow \mathcal{O}(Q P)
$$

indeed, for each $\vec{f} \in \overrightarrow{\mathcal{F}}_{\mathcal{W}}$ we have induced maps

$$
P_{\vec{f}}:(Q P)^{-1} \vec{f} \rightarrow Q^{-1} \vec{f}
$$

and we can define our map as follows

$$
\mathcal{O}(P) \otimes \mathcal{O}(Q)=\bigotimes_{\vec{f} \in \overrightarrow{\mathcal{F}}_{\mathcal{W}}}\left[\mathcal{O}\left(P_{\vec{f}}\right) \otimes \mathcal{O}\left(Q^{-1} \vec{f}\right)\right] \rightarrow \underset{\vec{f} \in \overrightarrow{\mathcal{F}}_{\mathcal{W}}}{\bigotimes} \mathcal{O}\left((Q P)^{-1} \vec{f}\right)=\mathcal{O}(Q P)
$$

The property then reads that the maps $\circ(Q, P)$ should be associative in the obvious way.

5.2.2 It is immediate that the complexes $\operatorname{full}_{\mathcal{K}}\left(U^{\prime}\right)$ form a colored 2 -operad full $_{\mathcal{K}}$.

5.2.3 Given a Chrom-colored 2-operad $\mathcal{O}$, we define an $\mathcal{O}$-algebra as an Chrom-family $\mathcal{K}$ of $\mathcal{C}$-complexes along with a map of colored 2-operads

$$
\mathcal{O} \rightarrow \text { full }_{\mathcal{K}}
$$

5.2.4 Define a (non-colored) 2-operad as a colored operad with the set of colors that have only one element. ${ }^{4}$

\subsection{Main theorem}

DG-categories and their functors form a category. Fix a small sub-category $\mathcal{C}$ in this category. Given a globe $g$ in $\mathcal{C}$, we have defined a complex $\operatorname{Rhom}(g)$. These complexes form a $\mathcal{C}$-complex, to be denoted Rhom. Likewise we have the functor of usual homomorphisms hom $(g)$, these also form a $\mathcal{C}$-complex hom. We have a natural map of $\mathcal{C}$-complexes

$$
\text { hom } \rightarrow \text { Rhom. }
$$

We know that the pair $(C$, hom $)$ is naturally a 2-category. This can be formulated in our language as follows. Define a trivial 2-operad triv as follows: $\operatorname{set} \operatorname{triv}(\mathcal{U})=k$ for each 2-ordinal $\mathcal{U}$ and demand that all structure maps preserve $1 \in k$. Then the 2 -categorical structure on $(\mathcal{C}$, hom $)$ amounts to the fact that we have a triv-algebra structure on hom.

5.3.1 Formulation of a theorem. Define a notion of a contractible 2-operad as a collection of the following data:

(i) a 2-operad $\mathcal{O}$;

(ii) a quasi-isomorphism of 2-operads $\mathcal{O} \rightarrow$ triv.

Theorem 5.1. There exists a contractible 2-operad $\mathcal{O}$ and $\mathcal{O}$-algebra structures on hom and Rhom such that:

(i) the map hom $\rightarrow$ Rhom is a map of $\mathcal{O}$-algebras;

(ii) the $\mathcal{O}$-algebra structure on hom is the pull-back of the triv-structure via the structure map $\mathcal{O} \rightarrow$ triv.

\footnotetext{
${ }^{4}$ This definition is narrower than Batanin's: in his papers, such objects are called '1-terminal 2-operads'; however, all 2-operads in our paper are 1-terminal.
} 


\section{TAMARKIN}

The rest of the paper is devoted to proving this theorem.

\section{Constructing a colored 2-operad which acts on hom}

Let $\mathbb{N}$ be the set of isomorphism classes of ordinals, $\mathbb{N}=\{[0],[1],[2], \ldots\}$. We then have an $\mathbb{N}$-family of $\mathcal{C}$-complexes $I \mapsto \mathbf{h o m}^{I}, I \in \mathbb{N}$.

We start with the construction of a colored 2-operad seq in the category of sets acting naturally ${ }^{5}$ on hom $^{\bullet}$. (Note that in the definition of a colored operad in $\S 5.2 .1$, the category of complexes can be replaced with any symmetric monoidal category.)

By default, all colorings are $\mathbb{N}$-colorings.

\subsection{Construction of seq}

Let $(\mathcal{U}, \chi)$ be a colored 2-ordinal. Let $\pi: \overrightarrow{\mathcal{F}}_{\mathcal{U}} \rightarrow \overrightarrow{\mathcal{C}}_{\mathcal{U}}$ be the induced 2-tree.

Let us use the following notation for the ordinals which determine the coloring:

$$
\begin{aligned}
I_{\vec{f}} & :=c_{\vec{f}}^{\chi} ; \\
J & :=c^{\chi} .
\end{aligned}
$$

Define a set $\mathbf{s e q}(\mathcal{U})$ each of the elements of which is a collection of the following data:

(a) a total order on $\mathcal{I}:=\mathcal{I}_{\mathcal{U}}:=\coprod_{\vec{f} \in \overrightarrow{\mathcal{F}}} I_{\vec{f}}$;

(b) a monotone map $W: \mathcal{I} \rightarrow J$.

The conditions are:

(i) the total order on $\mathcal{I}$ agrees with the orders on each $I_{f}$;

(ii) if $i_{1}, i_{2} \in I_{\vec{f}}$ and $i_{1}<i<i_{2}, i \in I_{\vec{f}_{1}}$, then $\pi\left(\vec{f}_{1}\right)<\pi(\vec{f})$;

(iii) if $\pi\left(\vec{f}_{1}\right)=\pi\left(\vec{f}_{2}\right)=\vec{c}$ and $\vec{f}_{1}<\vec{f}_{2}$ in the sense of the order on $\overrightarrow{\mathcal{F}}_{\vec{c}}$, then $I_{\vec{f}_{1}}<I_{\vec{f}_{2}}$ with respect to the order on $\mathcal{I}$.

6.1.1 Compositions. Let $P: \mathcal{U}^{\prime} \rightarrow \mathcal{V}^{\prime}$ be a map of $\mathbb{N}$-colored ordinals. Define the structure map

$$
\circ_{p}: \prod_{\vec{f} \in \overrightarrow{\mathcal{F}}_{\mathcal{V}}} \operatorname{seq}\left(P^{-1} \vec{f}\right) \times \operatorname{seq}(\mathcal{V}) \rightarrow \operatorname{seq}(\mathcal{U}) .
$$

Let us pick elements $\lambda_{\vec{f}} \in \mathbf{s e q}\left(P^{-1} \vec{f}\right), \lambda \in \mathbf{s e q}(V)$ and define their composition. We have

$$
\mathcal{I}_{\mathcal{U}}=\coprod_{\vec{f} \in \overrightarrow{\mathcal{F}}_{\mathcal{V}}} \mathcal{I}_{P^{-1} \vec{f}}
$$

The elements $\lambda_{\phi}$ define total order on $\mathcal{I}_{P^{-1} \vec{f}}$ and monotone maps

$$
\mathcal{I}_{P^{-1} \rightarrow} \rightarrow I_{\vec{f}}
$$

\footnotetext{
${ }^{5}$ We have a functor of taking the span, from the category of sets to the category of vector spaces. Treating vector spaces as complexes sitting in degree 0, we obtain a symmetric monoidal functor from the category of sets to the category of complexes. The latter functor induces a functor from the category of colored 2-operads in sets to the category of 2 -operads in the category of complexes. Let $k[\mathbf{s e q}]$ be the colored operad in the category of complexes obtained by applying this functor to seq. Whenever we have a $\mathcal{C}$-complex $\mathcal{K}$ and a $k$ [seq]-algebra structure on $\mathcal{K}$, we will say that seq acts on $\mathcal{K}$.
} 
The element $\lambda$ defines a total order on

$$
\mathcal{I}_{\mathcal{V}}:=\coprod_{\vec{f}} I_{\vec{f}}
$$

We have a natural map

$$
M: \mathcal{I}_{\mathcal{U}}=\coprod_{\vec{f}} \mathcal{I}_{P^{-1} \vec{f}} \rightarrow \coprod_{\vec{f}} I_{\vec{f}}=I_{\mathcal{V}}
$$

Lemma 6.1. There is a unique total order on $\mathcal{I}_{\mathcal{U}}$ such that:

(i) the map $M$ is monotone;

(ii) this order agrees with those on each $\mathcal{I}_{P^{-1} \vec{f}}$;

Proof. If such an order exists, it must be defined as follows:

(i) if $x, y \in \mathcal{I}_{\mathcal{U}}$ and $M(x)<M(y)$, then $x<y$;

(ii) if $M(x)=M(y) \in I_{\phi}$, then $x, y \in \mathcal{I}_{P^{-1}} \vec{f}$.

It is clear that in this way we indeed get a total order on $\mathcal{I}_{\mathcal{U}}$. The map $M$ is automatically monotone. We only need to check the matching of this order with that on each $\mathcal{I}_{P^{-1}} \vec{f}$. This follows immediately from the monotonicity of the corresponding maps

$$
\mathcal{I}_{P^{-1} \rightarrow} \rightarrow I \rightarrow{ }_{f}
$$

Next we define a map $W^{\prime}: \mathcal{I}_{\mathcal{U}} \rightarrow J$ as a composition

$$
\mathcal{I}_{\mathcal{U}} \rightarrow \mathcal{I}_{\mathcal{V}} \stackrel{W}{\longrightarrow} J
$$

Lemma 6.2. The constructed order on $\mathcal{I}_{\mathcal{U}}$ and the constructed map $W^{\prime}$ give rise to an element in $\operatorname{seq}(\mathcal{U})$.

Proof. The proof is straightforward.

We define the composition of the elements $\lambda_{f}$ and $\lambda$ to be the constructed element in $\operatorname{seq}(\mathcal{U})$. One can check that this composition satisfies the associativity property.

Remark. There is an equivalent description of the 2-operad seq in terms of planar trees. A detailed account will appear in a forthcoming paper by the author, V. Dolgushev and B. Tsygan.

\subsection{Action of seq on Rhom}

We need a few auxiliary constructions.

6.2.1 Given a dg-category $A$, an ordinal $J$, and a map $X: J \rightarrow A$ we call any element in $A(X)$ (see the very beginning of the paper) a chain in $A$ or, more specificly, an $X$-chain in $A$. Fix a chain $h \in A(X)$.

Suppose that we are given an $X: J \rightarrow A$ as above. Suppose that, in addition, we are given an ordinal $R$ and an $R$-family of functors $F_{r}: A \rightarrow B$.

Next, for each $\vec{r}=\overrightarrow{r_{1} r_{2}} \in \vec{R}$, choose ordinals $I_{\vec{r}}$ and elements

$$
h_{\vec{r}} \in \operatorname{hom}^{I \vec{r}}\left(F_{r_{1}} ; F_{r_{2}}\right) .
$$




\section{TAMARKIN}

Finally let us fix a monotone map

$$
W: I=\coprod_{\vec{r} \in \vec{R}} I_{\vec{r}} \rightarrow J
$$

where the order on $I$ is defined by those on $\vec{R}$ and on each of $I_{\vec{r}}$.

Given all of these data, we construct a chain $c$ in $B$.

6.2.2 Let us now make a formal definition.

First of all we need to construct an ordinal $K$ and a map $Y: K \rightarrow B$ so that $c \in B(Y)$.

Constructing $K$. For $r \in R$, let $m_{r}$ be the supremum in $J$ of the set

$$
\coprod_{\overrightarrow{r_{1}} \underset{r_{2}}{ } \mid r_{2} \leqslant r} W\left(I_{\overrightarrow{r_{1}}} \overrightarrow{r_{2}}\right) \text {. }
$$

Let $M_{r}$ be the infimum in $J$ of the set

$$
\coprod_{\overrightarrow{r_{1} r_{2}} \mid r \leqslant r_{1}} W\left(I_{\overrightarrow{r_{1} r_{2}}}\right)
$$

We define

$$
K:=K(J, W):=\coprod_{r \in R}\left[m_{r}, M_{r}\right]
$$

We then have natural maps

$$
\begin{gathered}
\pi: K \rightarrow R, \\
\kappa: K \rightarrow J .
\end{gathered}
$$

Constructing a map $Y:=Y(X, W): K \rightarrow B$. Set

$$
Y\left(j_{r}\right)=F_{r}\left(\kappa\left(j_{r}\right)\right),
$$

where $j_{r} \in\left[m_{r} ; M_{r}\right] \subset K$.

Constructing the resulting chain $c \in B(Y)$. We will define a map

$$
\mu_{W}: A(X) \otimes \bigotimes_{\overrightarrow{r_{1}} \overrightarrow{r_{2}} \in \vec{R}} \operatorname{hom}^{I_{\overrightarrow{r_{1}}} \overrightarrow{r_{2}}}\left(F_{r_{1}} ; F_{r_{2}}\right) \rightarrow B(Y)
$$

so that

$$
c=\mu_{W}\left(h ;\left\{h_{\vec{r}}\right\}_{\vec{r} \in \vec{R}}\right) .
$$

For an interval $[a, b] \subset J$, let $X_{a b}:=\left.X\right|_{[a, b]}$.

Let $R=\{0<1<2<\cdots<N\}$. We then have

$$
A(X)=A\left(X_{m_{0} M_{0}}\right) \otimes A\left(X_{M_{0} m_{1}}\right) \otimes A\left(X_{m_{1} M_{1}}\right) \otimes \cdots .
$$

Let $W_{r, r+1}:=\left.W\right|_{I_{r, r+1}}$. We then have a dominant map

$$
W_{r, r+1}: I_{\overrightarrow{r, r+1}} \rightarrow\left[M_{r} ; m_{r+1}\right] .
$$

Set

$$
X_{r, r+1}^{\prime}: I_{r, r+1} \stackrel{W_{r, r+1}}{\longrightarrow}\left[M_{r} ; m_{r+1}\right] \stackrel{X}{\longrightarrow} A .
$$

Hence, we have an induced map

$$
W_{r, r+1}^{*}: A\left(X_{M_{r} ; m_{r+1}}\right) \rightarrow A\left(X_{r, r+1}^{\prime}\right) ;
$$


via substitution, we get a map

$$
A\left(X_{M_{r} m_{r+1}}\right) \otimes \mathbf{h o m}^{I \overrightarrow{r, r+1}}\left(F_{r} ; F_{r+1}\right) \rightarrow B\left(F_{r}\left(X\left(M_{r}\right)\right) ; F_{r+1}\left(X\left(m_{r+1}\right)\right)\right) .
$$

The functor $F_{r}$ induces a map

$$
A\left(X_{m_{r} M_{r}}\right) \rightarrow B\left(\left.Y\right|_{\left[m_{r} ; M_{r}\right]}\right)
$$

Combining these maps we get the desired map $\mu_{W}$. Let us consider an example in which

$$
\begin{gathered}
J=\left\{0_{j}<1_{j}<\cdots<10_{j}\right\}, \\
R=\{a<b<c\}, \\
I_{\overrightarrow{a b}}=\left\{0_{a}<1_{a}<2_{a}<3_{a}\right\}, \quad I_{\overrightarrow{b c}}=\left\{0_{b}<1_{b}<2_{b}\right\},
\end{gathered}
$$

and the map $W$ is given by the following table

$$
\begin{aligned}
0_{a} & \mapsto 1_{j}, \quad 1_{a}, 2_{a} \mapsto 3_{j}, \quad 3_{a} \mapsto 4_{j}, \\
0_{b} & \mapsto 5_{j}, \quad 1_{b} \mapsto 7_{j}, \quad 2_{b} \mapsto 8_{j} .
\end{aligned}
$$

Then our map is constructed according to the following picture.

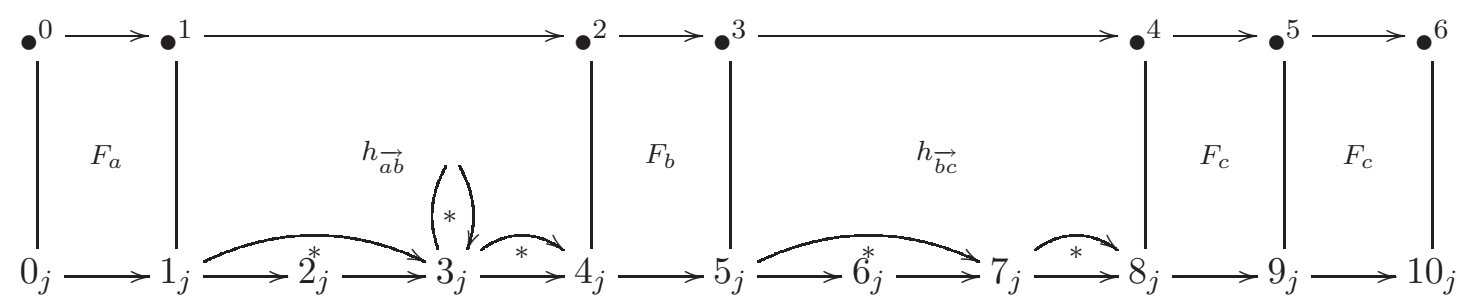

We explain the picture as follows:

(i) in each cell marked by $*$ we compose the arrows on the bottom of the cell;

(ii) in each cell marked by $h_{\overrightarrow{a b}}, h_{\overrightarrow{b c}}$ we apply the corresponding element from (9);

(iii) in each cell marked by $F_{a}, F_{b}$ or $F_{c}$ we apply the corresponding functor.

The resulting chain $c$ corresponds to the top line of the arrows in the picture.

Let us write an explicit formula for $c$. Fix a map $X: J \rightarrow A$ and a chain $f_{01} \otimes f_{12} \otimes \cdots \otimes f_{910} \in$ $A(X)$ so that $f_{k ; k+1}: X(k) \rightarrow X(k+1)$. The ordinal $K$ then corresponds to the fat points on the bottom line of the diagram, so $K=\{0<\cdots<6\}$. The map $Y: K \rightarrow B$ sends $\{0, \ldots, 6\}$ to, respectively,

$$
F_{a}(X(0)), \quad F_{a}(X(1)), \quad F_{b}(X(4)), \quad F_{b}(X(5)), \quad F_{c}(X(8)), \quad F_{c}(X(9)), \quad F_{c}(X(10)) .
$$

The resulting chain in $B(Y)$ is given by the following collection of arrows:

$$
F_{a}\left(f_{01}\right), \quad h_{\overrightarrow{a b}}\left(f_{23} f_{12} ; \operatorname{Id}_{X(3)} ; f_{34}\right), \quad F_{b}\left(f_{45}\right), \quad h_{\overrightarrow{b c}}\left(f_{67} f_{56} ; f_{78}\right), \quad F_{c}\left(f_{89}\right), \quad F_{c}\left(f_{9,10}\right) .
$$

6.2.3 Definition of a seq-algebra structure. Let us now construct the structure maps

$$
A: \operatorname{seq}(\mathcal{U}) \rightarrow \operatorname{full}_{\mathrm{hom}} \bullet(\mathcal{U}) .
$$

Equivalently, for each $\mathcal{U}$-diagram in $\mathcal{C}$, one has to construct a map

$$
k[\operatorname{seq}(\mathcal{U})] \otimes \bigotimes_{\vec{f} \in \overrightarrow{\mathcal{F}}} \operatorname{hom}^{I \vec{f}}\left(\left.\mathcal{D}\right|_{\vec{f}}\right) \rightarrow \operatorname{hom}^{J}\left(p_{*} \mathcal{D}\right),
$$

where $\left.\mathcal{D}\right|_{f}$ is the $\mathcal{C}$-globe obtained by the restriction of $\mathcal{D}$ onto $\vec{f}$ and $p_{*} \mathcal{D}$ is a $\mathcal{C}$-globe obtained by pre-composing $\mathcal{D}$ with the terminal map $p: \mathcal{U} \rightarrow$ globe. 


\section{TAMARKIN}

Let $\mu$ be the minimal element in $\overrightarrow{\mathcal{C}}$. Consider the ordinals $I_{\vec{f}}, \vec{f} \in \overrightarrow{\mathcal{F}}_{\mu}$. It follows that they form intervals in $\mathcal{I}$ and $\vec{f}_{1}<\vec{f}_{2}$ implies $I_{\vec{f}_{1}}<I_{\vec{f}_{2}}$ in $\mathcal{I}$.

We have a restriction

$$
W: \coprod_{\vec{f} \in \overrightarrow{\mathcal{F}}_{\mu}} I_{\vec{f}} \rightarrow J
$$

satisfying all of the conditions of the previous section. Hence, we have a map $\mu_{W}$ as explained above. Let us show that after the application of $\mu_{W}$, the remaining ingredients form a similar structure to that with which we started.

The map $\mu_{W}$ only involves the complexes hom ${ }^{I} \vec{f}$ with $\vec{f} \in \overrightarrow{\mathcal{F}}_{\mu}$. The remaining complexes are labeled by the elements of the set

$$
\mathcal{I}^{\prime}:=\mathcal{I} \backslash \coprod_{\vec{f} \in \overrightarrow{\mathcal{F}}_{\mu}} I_{\vec{f}}
$$

The map $W$ naturally descends to a map

$$
W^{\prime}: \mathcal{I}^{\prime} \rightarrow K(J, W)
$$

Let $\mathcal{C}^{\prime}:=\mathcal{C} \backslash m_{\mathcal{C}}$; we then get a diagram $\mathcal{U}^{\prime}$ with $\mathcal{C}_{\mathcal{U}^{\prime}}=\mathcal{C}^{\prime}$ and $\mathcal{F}_{\mathcal{U}^{\prime}, \vec{c}}=\mathcal{F}_{\mathcal{U}, \vec{c}}$. It then follows that $\left(\mathcal{I}^{\prime}, W^{\prime}\right) \in \operatorname{seq}\left(\mathcal{U}^{\prime}\right)$. Thus, we have constructed a map

$$
\nu \mathcal{U}: k[\operatorname{seq}(\mathcal{U})] \otimes \bigotimes_{\vec{f} \in \overrightarrow{\mathcal{F}}} \operatorname{hom}^{I \vec{f}}\left(\left.\mathcal{D}\right|_{\vec{f}}\right) \rightarrow k\left[\operatorname{seq}\left(\mathcal{U}^{\prime}\right)\right] \otimes \bigotimes_{\vec{f}^{\prime} \in \overrightarrow{\mathcal{F}}_{\mathcal{U}^{\prime}}} \operatorname{hom}^{I_{\vec{f}^{\prime}}}\left(F_{\left.P_{*} \mathcal{D}\right|_{\vec{f}^{\prime}}}\right) .
$$

One can now iterate this procedure, thus exhausting all of the arguments; in the end we will obtain a chain of morphisms in $C_{M_{\mathcal{C}}}$, and, finally, we can take the composition of all morphisms in this chain, which will produce the result.

We omit the proof that this is indeed a seq-algebra structure: this is pretty clear.

\subsubsection{Examples. Consider a pair of typical examples.}

Example 1. The 2-ordinal $U$ (see (10)) is as follows: $\mathcal{C}_{U}:=\{0<1\}, \mathcal{F}_{\overrightarrow{01}}=\left\{f_{0}<f_{1}<f_{2}\right\}$. We have $\mathcal{F}_{\mathcal{U}}:=\left\{\overrightarrow{f_{0} f_{1}}, \overrightarrow{f_{1} f_{2}}\right\}$

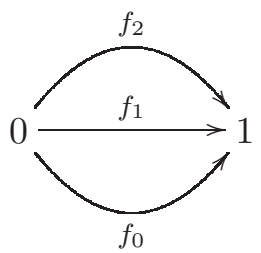

Fix a $\mathcal{U}$-diagram $\mathcal{D}$ so that we have dg-categories $C_{0}, C_{1}$ and functors $F_{0}, F_{1}, F_{2}: C_{0} \rightarrow C_{1}$. Let $I_{\overrightarrow{f_{0} f_{1}}}:=\left\{0_{f}<\cdots<n_{f}\right\}, I_{\overrightarrow{f_{1} f_{2}}}:=\left\{0_{g}<\cdots<m_{g}\right\}$, and $J:=\left\{0_{j}<\cdots<(n+m)_{j}\right\}$. Thus, we have a coloring of $\mathcal{U}$.

Fix the order on $\mathcal{I}:=I_{f} \sqcup I_{g}$ by prescribing $I_{f}<I_{g}$. Set

$$
W: I_{f} \sqcup I_{g} \rightarrow J
$$

as follows:

$$
W\left(p_{i}\right):=p_{j}, \quad W\left(q_{j}\right):=(n+q)_{j} .
$$

The order on $\mathcal{I}$ and the map $W$ determine an element $c \in \operatorname{seq}(\mathcal{U})$. This element determines an operation

$$
m_{c}: C^{I_{f_{0} f_{1}}}\left(F_{0} ; F_{1}\right) \otimes C^{I_{f_{1} f_{2}}}\left(F_{1} ; F_{2}\right) \rightarrow C^{J}\left(F_{0}, F_{2}\right) .
$$




\section{WHAT DO DG-CATEGORIES FORM?}

By definition, this operation is as follows.

Fix elements $\phi \in C^{I_{f_{0} f_{1}}}\left(F_{0} ; F_{1}\right), \psi \in C^{I_{f_{1} f_{2}}}\left(F_{1} ; F_{2}\right)$. Fix a chain $x$ in $C_{0}(J)$ by fixing objects $X_{s} \in C_{0}, 0 \leqslant s \leqslant n+m$ and morphisms $h_{s}: X_{s} \rightarrow X_{s+1}$. We set

$$
m_{c}(\phi, \psi)(x):=\psi\left(h_{n}, h_{n+1}, \ldots, h_{n+m-1}\right) \circ \phi\left(h_{0}, h_{1}, \ldots, h_{n-1}\right) .
$$

Example 2. Now let $\mathcal{U}$ (see (11)) be a 2-ordinal such that $\mathcal{C}_{\mathcal{U}}=\{0,1,2\}, \mathcal{F}_{\overrightarrow{01}}=\left\{f_{0}<f_{1}\right\}$, and globe $_{\overrightarrow{12}}:=\left\{g_{0}<g_{1}\right\}$. We then have $\mathcal{F}_{\mathcal{U}}=\left\{\overrightarrow{f_{0} f_{1}} ; \overrightarrow{g_{0} g_{1}}\right\}$. Denote $f:=\overrightarrow{f_{0} f_{1}} ; g:=\overrightarrow{g_{0} g_{1}}$ :

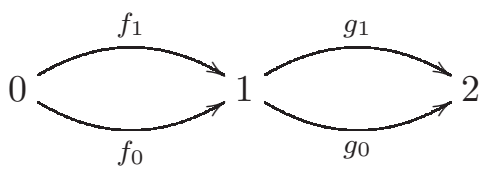

Fix a coloring of $\mathcal{U}$ by setting $I_{f}:=\left\{0_{f}<\cdots<n_{f}\right\}, I_{g}:=\left\{0_{g}<\cdots<m_{g}\right\}$. Let $J:=\left\{0_{j}<\right.$ $\left.\cdots<(n+m-1)_{j}\right\}$. Given a number $R \in\{1, \ldots, m\}$, let us define an element $s:=s_{R} \in \operatorname{seq}(\mathcal{U})$ by:

(i) fixing a total order on $\mathcal{I}$

$$
\left\{0_{g}<1_{g}<\cdots<(R-1)_{g}<0_{f} \cdots<n_{f}<R_{g}<(R+1)_{g}<\cdots<m_{g}\right\} ;
$$

(ii) fixing a map $W: \mathcal{I} \rightarrow J$ as a unique monotone surjective map such that $W\left((R-1)_{g}\right)=W\left(0_{f}\right)$ and $W\left(n_{f}\right)=W\left(R_{g}\right)$; more specifically,

$$
\begin{gathered}
0_{g} \mapsto 0_{j}, \quad 1_{g} \mapsto 1_{j}, \ldots,(R-1)_{g} \mapsto(R-1)_{j}, \\
0_{f} \mapsto(R-1)_{j}, \quad 1_{f} \mapsto R_{j}, \ldots, n_{f} \mapsto(R-1+n)_{j}, \\
R_{g} \mapsto(R-1+n)_{j}, \quad(R+1)_{g} \mapsto(R+n)_{j}, \ldots, m_{g} \mapsto(m-1+n)_{j} .
\end{gathered}
$$

Let us now fix a $\mathcal{U}$-diagram, i.e. categories $C_{0}, C_{1}, C_{2}$ and functors $F_{0}, F_{1}: C_{0} \rightarrow C_{1}$ and $G_{0}, G_{1}: C_{1} \rightarrow C_{2}$. Let us construct the operation

$$
m_{s_{R}}: \operatorname{hom}^{I_{f}}\left(F_{0}, F_{1}\right) \otimes \operatorname{hom}^{I_{g}}\left(G_{0}, G_{1}\right) \rightarrow \operatorname{hom}^{J}\left(G_{0} F_{0}, G_{1} F_{1}\right) .
$$

Let $h_{F} \in \operatorname{hom}^{I_{f}}\left(F_{0}, F_{1}\right)$ and $h_{G} \in \operatorname{hom}^{I_{g}}\left(G_{0}, G_{1}\right)$ and let $X_{0}, \ldots, X_{n+m-1}, u_{k}: X_{k} \rightarrow X_{k+1}$ be a chain of objects and arrows in $C_{0}$. Let $X: J \rightarrow C_{0}: k_{j} \mapsto X_{k}$. We then have a chain $u:=u_{0} \otimes \cdots \otimes u_{n+m-2} \in C_{0}(X)$. Let us construct the arrow

$$
m_{s_{R}}\left(h_{F}, h_{G}\right)(u): G_{0} F_{0}\left(X_{0}\right) \rightarrow G_{1} F_{1}\left(X_{n+m-1}\right)
$$

in $C_{2}$.

Applying $h_{F}$, we get the following chain in $C_{1}$ :

$$
\begin{aligned}
F_{0}\left(X_{0}\right) \stackrel{F_{0}\left(u_{0}\right)}{\longrightarrow} F_{0}\left(X_{1}\right) \stackrel{F_{0}\left(u_{1}\right)}{\longrightarrow} & \cdots \stackrel{F_{0}\left(u_{R-2}\right)}{\longrightarrow} F_{0}\left(X_{R-1}\right) \stackrel{h_{F}\left(u_{R-1}, u_{R}, \ldots, u_{R-2+n}\right)}{\longrightarrow} F_{1}\left(X_{R-1+n}\right) \\
& \stackrel{F_{1}\left(u_{R-1+n}\right)}{\longrightarrow} F_{1}\left(X_{R+n}\right) \stackrel{F_{1}\left(u_{R+n}\right)}{\longrightarrow} \cdots \stackrel{F_{1}\left(u_{R+n+m-2}\right)}{\longrightarrow} F_{1}\left(X_{n+m-1}\right) .
\end{aligned}
$$

We apply $h_{G}$ to this chain so as to get an arrow

$$
h_{G}\left(F_{0}\left(u_{0}\right) ; F_{0}\left(u_{1}\right) ; \ldots ; F_{0}\left(u_{R-2}\right) ; h_{F}\left(u_{R-1}, u_{R}, \ldots, u_{R-2+n}\right) ; F_{1}\left(u_{R-1+n}\right) ; \ldots ; F_{1}\left(u_{R+n+m-2}\right)\right)
$$

which belongs to

$$
\operatorname{hom}_{C_{2}}\left(G_{0} F_{0}\left(X_{0}\right) ; G_{1} F_{1}\left(X_{n+m-1}\right)\right) .
$$

This arrow is $m_{s_{R}}\left(h_{F}, h_{G}\right)(u)$.

6.2.5 In $\S 3.1 .1$ we have defined a map of cosimplicial complexes

$$
\operatorname{hom}^{\bullet}(F, G) \rightarrow \operatorname{hom}^{\bullet}(F, G)
$$




\section{TAMARKIN}

for every pair of functors $F, G: A \rightarrow B$. In this way, we get a map of $\mathcal{C}$-complexes

$$
\mathrm{hom}^{\bullet} \rightarrow \mathrm{hom}^{\bullet} \text {. }
$$

It is easy to check that hom ${ }^{\bullet}$ is a seq-subalgebra of hom ${ }^{\bullet}$. Furthermore, given a 2-ordinal $\mathcal{U}$ and a $\mathcal{U}$-diagram $\mathcal{D}$, for every $e \in \operatorname{seq}(\mathcal{U})$, the structure map

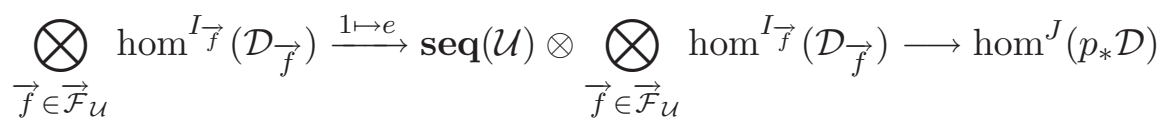

is the same.

This can be formulated as follows. Let $\mathcal{T}$ be the trivial $\mathbb{N}$-colored 2-operad: for every $\mathbb{N}$-colored 2-ordinal $\mathcal{U}, \mathcal{T}(\mathcal{U}):=\{1\}$, and the structure maps are then uniquely defined. Let

$$
\text { seq } \rightarrow \mathcal{T}
$$

be the obvious projection. We then have the following.

Proposition 6.3. We have:

(i) hom $^{\bullet} \subset$ hom $^{\bullet}$ is a seq-subalgebra;

(ii) the seq-action on hom $\bullet$ passes through the projection (12).

6.2.6 Cosimplicial structure. Let us recover the cosimplicial structure on hom ${ }^{\bullet}$ from the seqstructure.

Let globe, $I, J$ be the globe colored by the ordinals $I$ and $J$. By definition,

$$
\text { seq }(\text { globe }, I, J)=\Delta(I, J)
$$

is the space of all monotone maps.

The 2-operadic structure gives rise to associative maps

$$
\operatorname{seq}(\text { globe }, I, J) \times \operatorname{seq}(\text { globe }, J, K) \rightarrow \operatorname{seq}(\text { globe }, I, K)
$$

thus giving rise to a category structure on $\mathbb{N}$ which is just given by composing the corresponding monotone maps. That is, this category is nothing else but the simplicial category $\Delta$.

6.2.7 Given a colored 2-ordinal $\mathcal{U}^{\prime}$ with the underlying 2-ordinal $\mathcal{U}$ and a coloring given by the family of ordinals $I_{f}, \vec{f} \in \overrightarrow{\mathcal{F}}_{\mathcal{U}}, J$, write

$$
\operatorname{seq}(\mathcal{U})_{\left\{I_{\vec{f}}\right\}_{\vec{f} \in \overrightarrow{\mathcal{F}}}}^{J}:=\operatorname{seq}\left(\mathcal{U}^{\prime}\right)
$$

As a function in $I_{\vec{f}}, J$, seq $(\mathcal{U})$ becomes a functor

$$
\left(\Delta^{\mathrm{op}}\right)^{\overrightarrow{\mathcal{F}}} \times \Delta \rightarrow \text { sets }
$$

\subsection{Passing to complexes}

Define the realization

$$
\mathcal{O}(\mathcal{U}):=|\operatorname{seq}(\mathcal{U})|:=\operatorname{hom}_{\Delta}\left(\mathcal{S} ; k[\operatorname{seq}(\mathcal{U})] \otimes_{\left(\Delta^{\mathrm{op}}\right) \overrightarrow{\mathcal{F}}}(\mathcal{S})^{\otimes \overrightarrow{\mathcal{F}}}\right),
$$

where $\mathcal{S}$ is as in $\S 3.1$. A similar construction can be found in [MS04].

It is immediate that these realizations form a dg-2-operad $\mathcal{O}$, and that this operad acts on the $\mathcal{C}$-complex $\mathbf{R h o m}=\operatorname{hom}_{\Delta}\left(\mathcal{S}, \mathbf{h o m}^{\bullet}\right)$.

Our goal now is to check that this operad satisfies the theorem. 


\subsection{Contractibility of $\mathcal{O}$}

First of all, let us construct a quasi-isomorphism

$$
\mathcal{O} \rightarrow \text { triv } .
$$

It is easy to construct such a map: it is just the augmentation map. Let us show that this map is a quasi-isomorphism.

We start by studying a multisimplicial set

$$
\operatorname{seq}(\mathcal{U})_{\bullet, \ldots, \bullet}^{J}: \Delta^{F_{\mathcal{U}}} \rightarrow \text { sets },
$$

where $J$ is a fixed ordinal. Let $S(\mathcal{U}, J)$ be the topological space obtained by applying the standard topological realization functor to this multisimplicial set.

Proposition 6.4. For each ordinal $J$, the topological space $S(U, J)$ is contractible.

Proof. One can describe this realization explicitly. Let us so do: a point of $S(\mathcal{U}, J)$ is given by an equivalence class decompositions of a fixed segment $I:=I_{\mathcal{U}}$ of length $|\overrightarrow{\mathcal{F}}|$ into a number of subsegments labeled by the elements from $\overrightarrow{\mathcal{F}}_{\mathcal{U}}$.

The labeling should satisfy:

(a) if $\vec{f}_{1}, \vec{f}_{2} \in \overrightarrow{\mathcal{F}}$ and a segment labeled by $\vec{f}_{2}$ lies between those labeled by $\vec{f}_{1}$, then $\pi\left(\vec{f}_{1}\right)>$ $\pi\left(\vec{f}_{2}\right)$

(b) if $\pi\left(\vec{f}_{1}\right)=\pi\left(\vec{f}_{2}\right)=\vec{c}$ and $\vec{f}_{1}<\vec{f}_{2}$ in $\overrightarrow{\mathcal{F}} \vec{c}_{c}$, then all segments labeled by $\vec{f}_{1}$ are on the left-hand side of elements labeled by $\vec{f}_{2}$;

(c) the total length of all segments labeled by the same element $\vec{f}$ is 1 ;

(d) a monotone map $\vec{J} \rightarrow I$.

Two such points are equivalent if one is obtained from the other by a number of operations of the following two types:

(i) adding into or deleting from our decomposition a number of labeled segments of length 0;

(ii) joining two neighboring segments of our decomposition labeled by the same letter into one segment labeled by the same letter, or the inverse operation.

This space receives an obvious CW-structure. The proof that this space is indeed a realization is straightforward.

Let $S(\mathcal{U})$ be the space whose points are described by conditions (a)-(c) (without condition (d)), and the equivalence relation is the same. We then get an obvious isomorphism

$$
S(\mathcal{U}, J)=S(\mathcal{U}) \times \Delta^{J} .
$$

Remarks. We make the following remarks.

(1) One can prove that this is an isomorphism of cosimplicial spaces.

(2) The topological realization

$$
|\Sigma(\mathcal{U})|:=|S(\mathcal{U}, \bullet)|
$$

is then identified with the space $S(\mathcal{U}) \times R$, where $R$ is the space of monotone maps of a unit segment into the segment $I$. The spaces $\Sigma(\mathcal{U})$ form a topological 2-operad. This operad acts on topological realizations of the topological version of $\operatorname{hom}^{\bullet}(F, G)$.

Thus, it only remains to show that the space $S(\mathcal{U})$ is contractible. 


\section{TAMARKIN}

For simplicity, let us identify $\mathcal{C}$ with the set $0<\cdots<n$. Let $\mathcal{U}_{m}$ be a ball in $\mathcal{U}$ which is the preimage of $[m, n] \subset \mathcal{C}$. We then have a natural projection

$$
P_{m}: S\left(\mathcal{U}_{m}\right) \rightarrow S\left(\mathcal{U}_{m+1}\right) ;
$$

this projection sends a point in $S\left(\mathcal{U}_{m}\right)$ into a point obtained from it by collapsing each segment labeled by elements from $\pi^{-1} \overrightarrow{m, m+1}$ to a point.

Conversely, given:

(i) a point $x \in S\left(\mathcal{U}_{m+1}\right)$; and

(ii) a monotone map $U: \pi^{-1}(\overrightarrow{m, m+1}) \rightarrow I_{\mathcal{U}_{m+1}}$;

one can construct a point in $S\left(\mathcal{U}_{m}\right)$ by inserting unit segments labeled by $i \in \pi^{-1}(\overrightarrow{m, m+1})$ in place of the point $U(i)$.

It is clear that in this way we obtain a bijection

$$
S\left(\mathcal{U}_{m}\right) \cong S\left(U_{m+1}\right) \times \Delta^{\pi^{-1} m} .
$$

This argument implies that the space $S(\mathcal{U})$ is homeomorphic to a product of simplices and, hence, is contractible.

Remark. This proof is similar to McClure and Smith's proof of contractibility of cells of their operad $D_{2}$ (see [MS04]).

6.4.1 Let us now use Proposition 6.4 in order to show that the augmentation map $\mathcal{O} \rightarrow$ triv is a weak equivalence of dg-operads.

Consider the complex

$$
\Sigma(\mathcal{U}, J):=k\left[\operatorname{seq}(\mathcal{U})_{\bullet, \ldots, \bullet}^{J}\right] \otimes_{\Delta^{\mathcal{H}} \mathcal{U}} \mathcal{S}^{\otimes \mathcal{F}_{\mathcal{U}}},
$$

which is the realization of the multisimplicial complex $k\left[\operatorname{seq}(\mathcal{U})_{\bullet}^{J}, \ldots, \bullet \bullet\right.$.

Let $\mathbf{p} t:\left(\Delta^{\mathrm{op}}\right)^{\mathcal{F}_{\mathcal{U}}} \rightarrow$ sets be the constant functor sending everything to the fixed one-element set. We then have a natural augmentation map

$$
k\left[\operatorname{seq}(\mathcal{U})_{\bullet, \ldots, \bullet}^{J}\right] \rightarrow k[\mathbf{p} t] .
$$

The total complex of $k[\mathbf{p} t]$ is isomorphic to $k$, and we have an induced map

$$
\text { aug : } \Sigma(\mathcal{U}, J) \rightarrow k \text {. }
$$

Proposition 6.4 readily implies the following.

Corollary 6.5. The map aug is a quasi-isomorphism of complexes.

It is clear that $J \mapsto \Sigma(\mathcal{U}, J)$ is a cosimplicial complex. Let $k^{\prime}: \Delta \rightarrow$ complexes be the cosimplicial complex sending every object to $k$ and every arrow to the identity of $k$. It is straightforward to check that the map aug gives rise to a map of cosimplicial complexes

$$
\operatorname{aug}^{\prime}: \Sigma(\mathcal{U}, \bullet) \rightarrow k^{\prime}
$$

which is a term-wise quasi-isomorpism.

Taking the realization of the cosimplicial complexes on both sides, we get the augmentation map

$$
\mathcal{O}(\mathcal{U}) \rightarrow k
$$

which is automatically a quasi-isomorphism (because the realization functor preserves quasiisomorphisms). This implies the following.

COROLlary 6.6. The augmentation map $\mathcal{O} \rightarrow$ triv is a weak equivalence of $\mathrm{dg} 2$-operads. 


\section{WHAT DO DG-CATEGORIES FORM?}

6.4.2 Thus, we have proven the assertion of Theorem 5.1 that a contractible 2-operad acts on Rhom. The remaining conditions (i) and (ii) follow immediately from Proposition 6.3. This completes the proof of the theorem.

\section{Relation to Deligne's conjecture}

Given a dg-category $\mathcal{A}$, we can consider a complex $\operatorname{Rhom}\left(\operatorname{Id}_{\mathcal{A}}, \operatorname{Id}_{\mathcal{A}}\right)$. This complex is called the Hochschild complex of the category $\mathcal{A}$. If the category $\mathcal{A}$ has only one object $p$, then its Hochschild complex coincides with that of the associative algebra $\operatorname{End}_{\mathcal{A}}(p)$.

Thus, we denote

$$
\operatorname{Hoch}_{\mathcal{A}}:=\operatorname{Rhom}\left(\operatorname{Id}_{\mathcal{A}}, \operatorname{Id}_{\mathcal{A}}\right) .
$$

As a corollary of the just proven theorem, we have a certain structure on $\mathbf{H o c h}_{\mathcal{A}}$; before defining it, let us give it a name 'an $\mathcal{O}$-algebra structure on $\mathbf{H o c h}_{\mathcal{A}}$ '.

The definition is as follows. Given a complex $\mathcal{K}$ (for example, $\mathcal{K}:=\mathbf{H o c h}_{\mathcal{A}}$ ) we define a 2-operad full $_{\mathcal{K}}$ by setting

$$
\operatorname{full}_{\mathcal{K}}(\mathcal{U}):=\operatorname{hom}_{k}\left(\mathcal{K}^{\otimes \mathcal{F}_{\mathcal{U}}} ; \mathcal{K}\right)
$$

with the obvious insertion maps.

Remark. Of course, this construction is a particular case of the full 2-operad of a $\mathcal{C}$-complex, where $\mathcal{C}$ is the category with one object and one arrow so that there is only one globe in $\mathcal{C}$, and a $\mathcal{C}$-complex is the same as a usual complex, so that our complex $\mathcal{K}$ gives rise to a $\mathcal{C}$-complex and we can apply the construction of the full operad of a $\mathcal{C}$-complex. In this way, we get another construction of full $_{\mathcal{K}}$.

Given a 2-operad $\mathcal{E}$, we define an $\mathcal{E}$-algebra structure on $\mathcal{K}$ as a map of 2-operads

$$
E \rightarrow \text { full }_{\mathcal{K}}
$$

Theorem 5.1 immediately implies the following.

Proposition 7.1. As in the statement of the theorem, $\mathbf{H o c h}_{\mathcal{A}}$ has a structure of algebra over the 2-operad $\mathcal{O}$.

As $\mathcal{O}$ is a contractible 2-operad, a result from [Bat07] implies the following.

Corollary 7.2. A certain operad which is homotopy equivalent to the chain operad of little disks acts on $\operatorname{Hoch}_{\mathcal{A}}$.

This corollary is known as Deligne's conjecture on Hochschild cochains.

\section{ACKNOWLEDGEMENTS}

I would like to thank A. Beilinson for the statement of the problem and M. Batanin for explaining his results to me. I greatly appreciate numerous discussions with E. Getzler as well as his help with $\mathrm{T}_{\mathrm{E}} \mathrm{X}$. Special thanks are also due to the referee for his very helpful and thoroughly written report. 


\section{WHAT DO DG-CATEGORIES FORM?}

\section{REFERENCES}

Bat98 M. A. Batanin, Monoidal globular categories as a natural environment for the theory of weak n-categories, Adv. Math. 136 (1998), 39-103.

Bat07 M. A. Batanin, Symmetrisation of n-operads and compactification of real configuration spaces, Adv. Math. 211 (2007), 684-725.

Ber02 C. Berger, A cellular nerve for higher categories, Adv. Math. 169 (2002), 118-175.

BF04 C. Berger and B. Fresse, Combinatorial operad actions on cochains, Math. Proc. Cambridge Philos. Soc. 137 (2004), 135-174.

Dri04 V. Drinfeld, DG quotients of DG categories, J. Algebra 272 (2004), 643-691.

Joy97 A. Joyal, Disks, duality, and $\theta$-categories, Preprint (1997).

KS00 M. Kontsevich and Y. Soibelman, Deformations of algebras over operads and the Deligne conjecture, in Proc. Conf. Moshé Flato 1999, Dijon, Vol. I, Mathematical Physics Studies, vol. 21 (Kluwer, Dordrecht, 2000), 255-307.

MS02 J. E. McClure and J. H. Smith, A solution of Deligne's Hochschild cohomology conjecture, in Recent progress in homotopy theory, Baltimore, MD, 2000, Contemporary Mathematics, vol. 293 (American Mathematical Society, Providence, RI, 2002), 153-193.

MS04 J. E. McClure and J. H. Smith, Cosimplicial objects and little n-cubes. I, Amer. J. Math. 126 (2004), 1109-1153.

Str00 R. Street, The petit topos of globular sets, in Category theory and its applications, Montreal, QC, 1997, J. Pure Appl. Algebra 154 (2000), 299-315.

Vor00 A. A. Voronov, Homotopy Gerstenhaber algebras, in Proc. Conf. Moshé Flato 1999, Dijon, Vol. II, Mathematical Physics Studies, vol. 22 (Kluwer, Dordrecht, 2000), 307-331.

Dmitry Tamarkin tamarkin@math.northwestern.edu

Department of Mathematics, Northwestern University, 2033 Sheridan Road, Evanston, IL 60208-2730, USA 